\title{
Hysteresis Loss in Poloidal Coils of the Large Helical Device*)
}

\author{
Kazuya TAKAHATA, Hirotaka CHIKARAISHI, Toshiyuki MITO and Shinsaku IMAGAWA \\ National Institute for Fusion Science, 322-6 Oroshi-cho, Toki 509-5292, Japan
}

(Received 22 December 2010 / Accepted 15 February 2011)

\begin{abstract}
Hysteresis loss in poloidal coils of the Large Helical Device (LHD) has been measured during single-pulse operation. The superconductors of the coils are Nb-Ti cable-in-conduit conductors (CICC) cooled by forcedflow supercritical helium. The loss was measured by monitoring the enthalpy increase of the helium coolant between the inlet and outlet. Although the hysteresis loss was extracted by extrapolating several data sets from pulse excitations with different sweep rates, the extrapolated loss was much larger than the estimation using the magnetic hysteresis of the conductor. The anomalous increase in the loss is likely due to inter-strand coupling loss with long time constants from the order of 10 to $1000 \mathrm{~s}$. The calculations show that the additional coupling loss behaves like a hysteresis loss.
\end{abstract}

(c) 2011 The Japan Society of Plasma Science and Nuclear Fusion Research

Keywords: cable-in-conduit conductor, hysteresis loss, coupling loss, LHD, superconducting magnet

DOI: $10.1585 /$ pfr.6.2405077

\section{Introduction}

The Large Helical Device (LHD) is a full superconducting experimental fusion apparatus [1]. The superconducting magnetic system consists of two types of superconducting coils, pool-cooled helical coils and forcedflow-cooled poloidal coils. For the poloidal coils, a cablein-conduit conductor (CICC) is used. A forced-flow of supercritical helium at 4.4-4.7 K circulates inside the conductor as a coolant. The poloidal coils have maintained stable operation for 13 years.

An important issue in designing superconducting coils for fusion reactors is estimating the AC loss. Measurements of AC loss in the LHD superconducting coils can provide valuable information, because the LHD is one of the few machines with large superconducting magnets. In 2008 , additional pulse power supplies were installed to enhance the output voltages of the power supplies for the LHD poloidal coils [2]. The enhancement enables AC loss measurements to be made during more rapid pulse operations.

AC loss consists of two components: hysteresis loss and coupling loss. Hysteresis loss is heat loss caused by magnetic hysteresis in superconducting materials. Coupling loss is heat loss caused by a shielding current through superconducting filaments and resistive connections, such as a copper matrix and contacts between strands. We have previously reported measurements of coupling loss [3,4]. In this paper, we present measurements of hysteresis loss.

The loss extrapolated to zero frequency is conventionally attributed to magnetic hysteresis, and the linear increase with frequency to coupling currents. The hysteresis

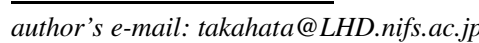

*) This article is based on the presentation at the 20th International Toki Conference (ITC20). loss in superconducting coils has actually been measured by extrapolating several pulse or cyclic excitation data sets with different sweep rates or frequencies. However, previous studies using other coils with CICC suggest that the extrapolated loss obtained by the above-mentioned method was much larger than the hysteresis loss estimated using the magnetic hysteresis of the strands or theoretical calculations [5,6]. Our measurements show similar behavior. In this paper, we investigate the reason for the difference between the extrapolated loss and the estimation of hysteresis loss.

\section{Measuring Procedure}

The poloidal coil system consists of three pairs of circular solenoids. The coils tested in this study were the smallest pair, the lower and upper inner vertical coils, IV$\mathrm{L}$ and IV-U. The average diameter of the coils is $3.6 \mathrm{~m}$. The two coils were connected to a power supply in series and were simultaneously excited with a single trapezoidal pulse. The trapezoidal waveform had the same upward and downward ramp times, $\tau_{0}$, and a flat top of $10 \mathrm{~s}, \tau_{1}$. The ramp times, $\tau_{0}$, were varied in the ranges $9-45 \mathrm{~s}$ for a maximum current of $2 \mathrm{kA}, 18-100 \mathrm{~s}$ for $4 \mathrm{kA}$ and $20-130 \mathrm{~s}$ for $5 \mathrm{kA}$.

The loss can be measured by monitoring the temperature and pressure at the inlet and outlet. The loss is always transferred to the coolant and the temperature of the coolant in the coil then increases. The coolant is always driven out from the outlet and the outlet coolant temperature then increases for a certain time. Therefore, the heat loss can be obtained by $m \Delta H$, where $m$ is the mass flow rate and $\Delta H$ is the enthalpy increase between the inlet and outlet [4]. 


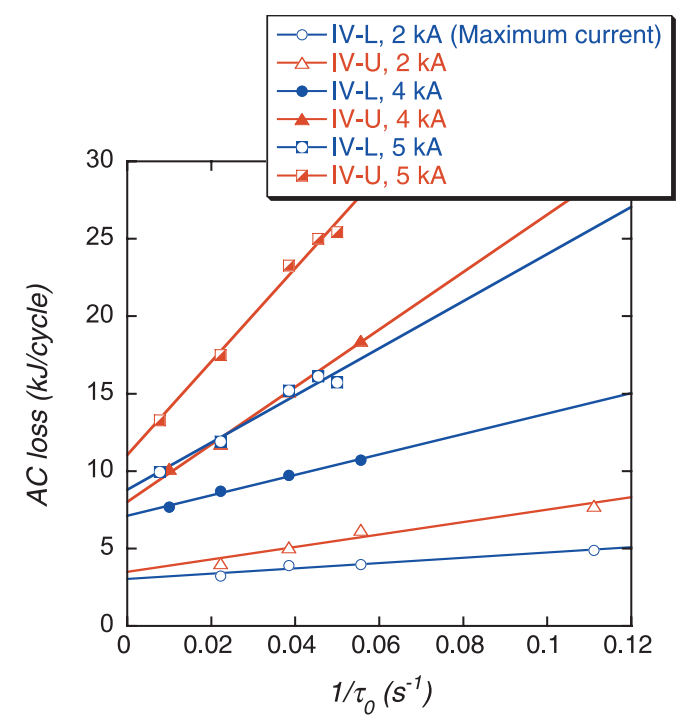

Fig. 1 Measured AC loss per pulse operation cycle as a function of the inverse of the ramp-up/down time.

\section{Results and Discussion}

Figure 1 shows the measured AC loss per pulse operation cycle as a function of the inverse of the ramp time, $1 / \tau_{0}$. The maximum current is fixed at 2,4 and $5 \mathrm{kA}$. The extrapolated loss was defined by extrapolating the slope of the data to $1 / \tau_{0}=0$ by linear fitting. The coupling loss can be extracted by subtracting the extrapolated loss from the measured loss. The time constants of the coupling loss were 0.08 and $0.2 \mathrm{~s}$ for the IV-L and IV-U coils, respectively [4].

We also estimated the hysteresis loss using the magnetic hysteresis in the conductor. We first measured static magnetization of a short conductor sample. Then, to calculate the loss as a function of the maximum current, we estimated parameters, such as the penetration field, and drew hysteresis loops using a theoretical model [7] as shown in Fig. 2. The area of the loop corresponds to the loss. Finally, the total loss in the coil was estimated as the sum of the losses in each turn, because each turn is subject to different magnetic fields.

Figure 3 shows the extrapolated and calculated hysteresis losses as a function of the maximum current. We found a clear difference between the extrapolated and hysteresis losses. This difference then increased with increasing maximum current. We suggest that the additional coupling loss due to inter-strand coupling currents with long loops behaves like a hysteresis loss. The current loops may have a wide range of coupling time constants, which are distributed over the order of 10-1000s [3].

In this study, we assume that the loops are formed at random, and the length of the loop is proportional to its time constant. Because the coupling current generates loss at the contact points between strands at both ends of the loop, the density of the contact points is inversely proportional to the time constant, $\tau$. Under these assumptions, the

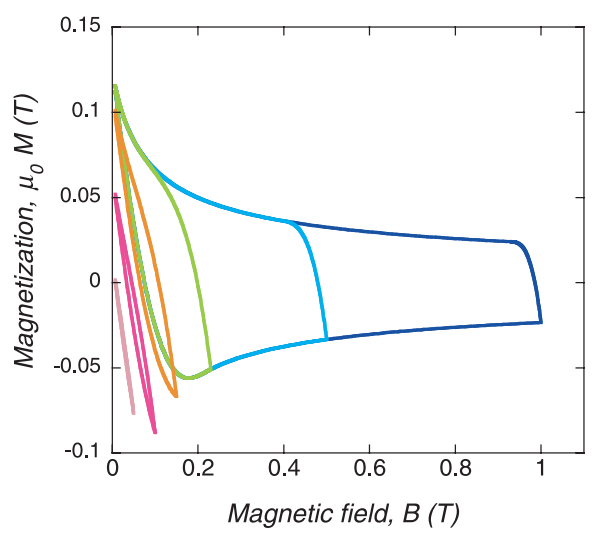

Fig. 2 Calculated magnetic hysteresis loops with different maximum fields.

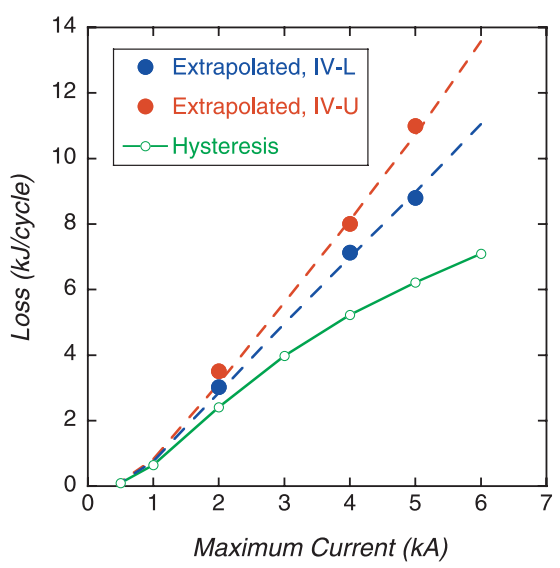

Fig. 3 Extrapolated and calculated hysteresis losses as a function of the maximum current.

additional coupling loss, $Q_{\text {add }}$ is given by

$$
Q_{\mathrm{add}}=\int_{\tau_{\mathrm{L} 1}}^{\tau_{\mathrm{L} 2}} \frac{\alpha}{\tau} Q_{\mathrm{c}}(\tau) \mathrm{d} \tau
$$

where $\tau_{\mathrm{L} 1}$ and $\tau_{\mathrm{L} 2}$ are the minimum and maximum of the $\tau$ distribution and $\alpha$ is a constant factor. According to the theoretical expression for a circuit model [8], $Q_{\mathrm{c}}$ can be given by

$$
\begin{aligned}
Q_{\mathrm{c}}= & \left(A^{*} B_{\mathrm{m}}^{2} V / \mu_{0}\right) \\
& \times v\left\{2+v\left(1-e^{-1 / v}\right)\left(e^{-(1 / v+1 / \kappa)}-e^{-1 / \kappa}-2\right)\right\},
\end{aligned}
$$

where $A^{*}$ is a dimensionless factor depending on the geometrical shape of the conductor cross section, $B_{\mathrm{m}}$ is the maximum field, $V$ is the volume of the strand and $\mu_{0}$ is the permeability. The dimensionless factors $v$ and $\kappa$ are defined as $v=\tau / \tau_{0}$ and $\kappa=\tau / \tau_{1}$. In the experiments, we set the flat top time, $\tau_{1}$, at $10 \mathrm{~s}$. $A^{*}$ and $B_{\mathrm{m}}$ depend on the position in the coil. Thus, we integrate $A^{*} B_{\mathrm{m}} V$ of each turn to evaluate the total loss in the coil.

Next, we demonstrate that the AC loss can be estimated using our assumption. Figure 4 shows the measured and calculated losses in the IV-U coil when the maximum 


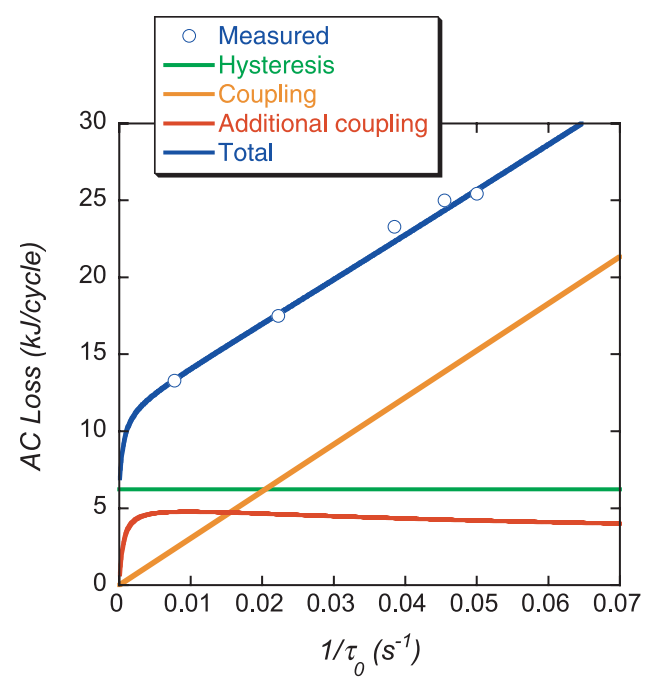

Fig. 4 Measured and calculated AC losses per pulse operation cycle in the IV-U coil when the maximum current is $5 \mathrm{kA}$.

current is $5 \mathrm{kA}$. We assume that $\alpha=0.0058, \tau_{\mathrm{L} 1}=10 \mathrm{~s}$ and $\tau_{\mathrm{L} 2}=1000 \mathrm{~s}$ for the calculation of Eq. (1). $\alpha$ was determined by fitting the calculation to the measured data. The calculated additional coupling loss is almost independent of the sweep time in the range 20 to $130 \mathrm{~s}$ in which we measured the data and behaves like a hysteresis loss. The total loss, the sum of the hysteresis, coupling and additional coupling losses, is thus consistent with the experimental data. The calculations imply that the additional coupling loss depends significantly on the sweep time when $\tau_{0}>\tau_{\mathrm{L} 2}$. Therefore, if AC losses are measured with a sweep time of around $\tau_{\mathrm{L} 2}$, the measured data may not lie in a straight line.

The dependence of the maximum current can also be explained qualitatively by Eq. (2). The additional coupling loss increases with increasing maximum current because the loss is proportional to the square of the maximum magnetic field, $B_{\mathrm{m}}^{2}$. In fact, we find a good fit with the measured data assuming that the additional coupling loss is proportional to the square of the maximum current as shown in Fig. 3. The fitting curves indicated by broken lines are consistent with the data.

To measure hysteresis loss more accurately, a periodic field variation, such as a sinusoidal wave with DC offset, is better than a single pulse according to a theoretical circuit model [8]. Because the loss with long time constants can be eliminated by a periodic variation, the difference between the extrapolated and hysteresis losses may decrease. In fact, hysteresis loss measurements with the KSTAR central solenoid model coils agree well with the estimation when using a sinusoidal wave [6]. We plan to operate the LHD poloidal coils with a periodic field variation and make hysteresis loss measurements.

\section{Conclusions}

Hysteresis loss was measured for two LHD poloidal coils with a CICC. However, the loss extrapolated to a zero sweep rate was much larger than the estimation from the magnetic hysteresis of the conductor. It is likely that the coupling currents with long time constants produce additional loss that behaves like a hysteresis loss. The time constants may be continuously distributed over the range of the order of 10-1000 s. When designing a large coil with a CICC, the estimation of additional coupling loss will become an increasingly important issue.

\section{Acknowledgements}

This work was supported in part by MEXT of the Japanese government under Grant NIFS10ULAA703 and NIFS10ULAA008. The authors wish to thank the members of the LHD device engineering group for their technical assistance.

[1] M. Fujiwara et al., J. Fusion Energy 15, 7 (1996).

[2] H. Chikaraishi et al., Proc. 13th European Conf. on Power Electronics and Applications, Barcelona, Spain, 2009, Article number 5279116.

[3] K. Takahata et al., Fusion Eng. Des. 65, 39 (2003).

[4] K. Takahata et al., IEEE Trans. Appl. Superconductivity 20, 517 (2010).

[5] Y. Takahashi et al., IEEE Trans. Appl. Superconductivity 11, 1546 (2001).

[6] S. Lee et al., IEEE Trans. Appl. Superconductivity 16, 771 (2006).

[7] K. Takahata et al., Fusion Eng. Des. 81, 2571 (2006).

[8] V. B. Zenkevitch and A. S. Romaniuk, IEEE Trans. Magn. MAG-13, 567 (1977). 